\title{
In Vitro Safety "Clinical Trial” of the Cardiac Liability of Hydroxychloroquine and Azithromycin as COVID19 Polytherapy
}

Bérénice Charrez ${ }^{1 \psi}$, Verena Charwat ${ }^{1 \psi}$, Brian Siemons ${ }^{1}$, Henrik Finsberg $^{3}$, Evan Miller ${ }^{2,5,6}$, Andrew G. Edwards $^{4}$, Kevin E. Healy ${ }^{1,7^{*}}$

1. Department of Bioengineering and California Institute for Quantitative Biosciences (QB3), University of California at Berkeley, Berkeley, California 94720, USA

2. Department of Chemistry, University of California at Berkeley, Berkeley, California 94720, USA

3. Simula Research Laboratory, 1325 Oslo, Norway

4. Department of Pharmacology, School of Medicine, University of California, Davis, Davis, CA 95616, USA

5. Department of Molecular and Cell Biology, University of California at Berkeley, Berkeley, California, 94720, USA

6. Helen Wills Neuroscience Institute, University of California, Berkeley, Berkeley, California, 94720, USA

7. Department of Materials Science and Engineering, University of California at Berkeley, Berkeley, California 94720, USA

$\psi$ Authors contributed equally to the work

* Corresponding author:

Kevin E. Healy, PhD

370 Hearst Memorial Mining Building, \# 1760

Berkeley, CA 94720

Phone: 510-643-3559

Email: kehealy@berkeley.edu

Conflict of interest: Professors Kevin E. Healy, Andy Edwards, Brian Siemons and Henrik Finsberg have financial relationships with Organos Inc. and both they and the company may benefit from the results of this research.

Funding Information: This work was funded in part by the California Institute for Regenerative Medicine DISC2-10090 (K.E.H.), NIH-NHLBI HL130417 (K.E.H.), NIH-NIGMS R35GM1195855 (E.W.M.) and the Jan Fandrianto and Selfia Halim Chair Fund (K.E.H.) 
bioRxiv preprint doi: https://doi.org/10.1101/2020.12.21.423869; this version posted December 28, 2020. The copyright holder for this preprint (which was not certified by peer review) is the author/funder. All rights reserved. No reuse allowed without permission.

Keywords: SARS-CoV-2, hydroxychloroquine, azithromycin, polytherapy, microphysiological systems, induced pluripotent stem cells, heart muscle, cardiomyocytes. 


\section{Abstract}

Despite global efforts, there are no effective FDA-approved medicines for the treatment of SARS-CoV-2 infection. Potential therapeutics focus on repurposed drugs, some with cardiac liabilities. Here we report on a preclinical drug screening platform, a cardiac microphysiological system (MPS), to assess cardiotoxicity associated with hydroxychloroquine (HCQ) and azithromycin (AZM) polytherapy in a mock clinical trial. The MPS contained human heart muscle derived from patient-specific induced pluripotent stem cells. The effect of drug response was measured using outputs that correlate with clinical measurements such as QT interval (action potential duration) and drug-biomarker pairing.

Chronic exposure to HCQ alone elicited early afterdepolarizations (EADs) and increased QT interval from day 6 onwards. AZM alone elicited an increase in QT interval from day 7 onwards and arrhythmias were observed at days 8 and 10. Monotherapy results closely mimicked clinical trial outcomes. Upon chronic exposure to HCQ and AZM polytherapy, we observed an increase in QT interval on days 4-8.. Interestingly, a decrease in arrhythmias and instabilities was observed in polytherapy relative to monotherapy, in concordance with published clinical trials. Furthermore, biomarkers, most of them measurable in patients' serum, were identified for negative effects of single drug or polytherapy on tissue contractile function, morphology, and antioxidant protection.

The cardiac MPS can predict clinical arrhythmias associated with QT prolongation and rhythm instabilities. This high content system can help clinicians design their trials, rapidly project cardiac outcomes, and define new monitoring biomarkers to accelerate access of patients to safe COVID-19 therapeutics. 


\section{Introduction}

When the World Health Organization declared a global pandemic on March 11th 2020, little was known about the pathogenesis of the severe acute respiratory syndrome coronavirus 2 (SARS-CoV-2). It was described and treated as a respiratory disease, in which the virus targeted the epithelial cells of the respiratory tract, resulting in alveolar damage, edema and fibrosis. Now, with more than 45 million cases and a million deaths worldwide, there is clinical evidence that the virus also has non-negligible long-term effects on multiple organs, including heart, kidney, vasculature, liver and even brain[1-7].

With the absence of FDA-approved medicines for the treatment or prevention of COVID-19, clinicians have been pressed to treat patients in critical stages without FDA approved protocols. They have therefore relied on several small scale clinical studies to repurpose compounds approved by regulatory bodies as monotherapies in the hope of improving patient outcomes. Early clinical trials identified chloroquine (CQ), hydroxychloroquine (HCQ) and azithromycin (AZM) as promising drugs to help treat or reduce the effects of SARS-CoV-2 [8-12]. A non-randomized clinical trial in France identified $\mathrm{HCQ}$, in combination with AZM, as being capable of significantly reducing respiratory viral loads [8]. This was confirmed by more recent retrospective studies[13, 14], but was also heavily criticized and refuted by other recent studies employing a priori designs[15-18]. Several additional small clinical trials have shown mixed outcomes for HCQ treatment of COVID-19 patients[19].

A serious concern with these studies is that patients were treated with drugs that have known cardiac complications, and their effects on the heart in polytherapy were unknown. HCQ inhibits $h E R G\left(\boldsymbol{I}_{\boldsymbol{K}}\right)$ potassium channels, it is known to increase in QT interval of cardiomyocytes, and can induce arrhythmias that are responsible for sudden death [20]. AZM is also associated with an increased risk of cardiovascular death, due to Torsade de Pointes (TdP) and polymorphic ventricular tachycardia [21]. With respect to polytherapy, clinical trials have demonstrated a synergistic effect of HCQ and AZM to prolong QT interval [22, 23]; however, alterations in arrhythmic event frequency were controversial when compared to HCQ or AZM alone. 
In the absence of rapid clinical trials for polytherapy safety, there is an urgent need for screening tools to increase the speed at which potential therapeutics are evaluated for cardiac liabilities. Common in vitro systems used for cardiac drug screening include cell 2D monolayers and animal testing, which often fail to replicate human physiology, particularly electrophysiology, and pharmacokinetic properties [24]. Engineered heart tissue, organoids or microfluidic-based microphysiological system are emerging alternatives for state-of-the-art drug screening $[25,26]$. With the rapid spread of COVID-19, microphysiological systems (MPS) have recently shown to be a promising tool to study virus entry and replication mechanisms, subsequent cytokine production, as well as effects of existing and novel therapeutics or vaccines $[27,28]$.

In this paper, we demonstrate the utility of a cardiac MPS (Figure 1) for determining the cardiac liability associated with HCQ and AZM polytherapy in an in vitro design analogous to a Phase I safety clinical trial. Our cardiac MPS contains a three-dimensional (3D) cell chamber in which human induced pluripotent stem cell-derived cardiomyocytes (hiPSC-CMs) are confined and self-assemble to form uniaxially beating heart muscle $[24,29]$. HiPSC-CMs have been successfully used for in vitro assessment of drug-induced arrhythmias, especially since they respond consistently to $h E R G$ channel block (QT prolongation and arrhythmias) and calcium channel block (action potential duration shortening, impaired contractile function) $[6,30]$. It makes them excellent candidates to screen for cardiac liability of HCQ and AZM, both of which are known to block hERG channels, and also act upon other cardiac ion channels[31]. By assaying hiPSC-CMs expressing a genetically encoded calcium sensor (GcAMP6f), loaded with a voltage-sensitive fluorescent probe (BeRST), we assessed the electrophysiology and calcium handling of the tissues during serial drug exposures. We showed that $\mathrm{HCQ}$ and $\mathrm{AZM}$ significantly increase $80 \%$ repolarization time $\left(\mathrm{APD}_{80}\right)$ and rhythm instabilities, starting at clinically relevant exposure days, and were accompanied with $E A D$ and TdP instances. $H C Q+A Z M$ combination also showed a significant increase in $A P D_{80}$, however, few instabilities or arrhythmic events were observed. Finally, proteomics analysis of cell culture effluent enabled detection of 
biomarkers that were directly correlated with cardiotoxicity, apoptosis and contraction mechanics alteration.

\section{Results and Discussion}

\section{Chronic exposure to $H C Q$ for 10 days resulted in QT prolongation and rhythm instabilities that} correlated with arrhythmic events and clinical observations

Chronic exposure to an $\mathrm{HCQ}$ dose mimicking clinical protocols decreased the beat rate starting at day 4 with a statistically signifcant decrease on days 4,7 and 8 (Figure 2A). APD 80 increased markedly from day 7 onwards (Figure 2B), with the maximum $\mathrm{APD}_{80}$ increase reaching $850 \mathrm{~ms}$. All but one tissue exhibited an $\mathrm{APD}_{80}$ at least 400 ms longer on day 7 and day 9 than prior to HCQ exposure. These arrhythmogenic changes to the AP were tightly correlated with the appearance of instabilities in the $\mathrm{HCQ}$ tissues from day 5 onwards (Figure $3 \mathrm{~A}$ ). $\mathrm{APD}_{80}$ was directly correlated to calcium transient duration (CaD) (Supp. Figure 1D), and an increase of $\mathrm{CaD}_{80}$ above $600 \mathrm{~ms}$ was observed at day 2 and increased over the duration of HCQ treatment. Arrhythmic events began appearing at day 4 in $50 \%$ of the tissues. At day 7, tissues exhibited both arrhythmogenic AP waveforms and $\mathrm{CaD}_{80}$ increase, and by days 9 and $10,50 \%$ of tissues exhibited weak or no dynamic signal change, suggesting loss of resting membrane potential (Figure 3D). Representative traces of arrhythmic events are shown in Figure 3 G$\mathbf{J}$, comparing 30 second calcium traces at day 0 and day 9 (Figure $\mathbf{3 G}$ ). Late calcium peaks are a signature of EADs in membrane potential and were clearly observed (red arrows) in day $9 \mathrm{HCQ}$ recordings, as were the marked increases in duration of the calcium transient, itself a marker for APD prolongation. Together these data reflect HCQ's well known block of $I_{K r}$, which prolongs the QT interval and its in vitro proxy APD [22, 32] which are associated with arrhythmic events[20, 33, 34]. Our AP data, which is consistant with the clinical literature, indicates that the cardiac MPS system is a good predictor of clinical cardiotoxicity of $\mathrm{HCQ}$. 


\section{Chronic exposure of AZM for 10 days showed QT prolongation and rhythm instabilities that} correlated with arrhythmic events and clinical observations

Chronic AZM exposure did not significantly change beat rate (Figure 2C). However, as for $\mathrm{HCQ}$, chronic $A Z M$ treatment increased $A P D_{80}$ by day 7 , and a trend for persisting prolonged $A P D_{80}$ continued until day 10, albeit with large variation (Figure 2D). Triangulation also trended $(p<0.2)$ to increase from day 8 onwards, although this did not reach significance ( $p=0.07$ at day 10) (Supp. Figure 1B). Instabilities arose at day 6 and worsened over time (Figure 3B). Non-parametric analysis of instability showed a significant increase of chaotic polygons in AZM-treated tissues when compared with $\mathrm{HCQ}\left(\mathrm{p}<0.05\right.$, not shown). $\mathrm{CaD}_{80}$ increased beyond $600 \mathrm{~ms}$ at day 4 , and day 6 onwards in $30-50 \%$ of the tissues. Arrhythmic events were observed from day 3 onwards, reaching $60 \%$ of tissues at day 10, with some tissues exhibiting both arrhythmia and $\mathrm{CaD}_{80}$ increase. Day 6 and 9 had respectively $16 \%$ and $33 \%$ of tissues with weak or no signal (Figure 3E). Figure 3 shows a representative trace of arrhythmic events at day 0 versus day 10 (Figure $3 \mathbf{H}$ ) of AZM exposure. Overall, $A \mathrm{PD}_{80}$ increased on all treatment days compared to day 0 , and EADs appeared after day 3 , and were most prevalent on day 10. Since clinical AZM application is typically limited to 5 days, the observed incidence of proarrhythmic events during longer exposure times cannot be directly compared to clinical outcomes. However, AZM has been associated with increase in cardiovascular death, mostly through QT prolongation and arrhythmia [21, 35], and these outcomes are clearly indicated by our MPS measurements.

\section{Chronic exposure to both HCQ and AZM for 10 days showed QT prolongation and rhythm instabilities that correlated with arrhythic events and clinical observations}

The beat rate increased at day 3 and 6 ; however, there was no clear overall trend (Figure 2E). APD 80 was significantly increased on days 5 and 8, with a trend towards APD prolongation for all recordings after day $7(\mathrm{p}<0.2)$ (Figure $\mathbf{2 F})$. This data set closely mimics clinical trials performed by Chorin et al. [36], where the QT interval increased starting at day 2 to day 5 with high variability in patient population. 
Triangulation was not significantly altered over the 10 days during our in vitro polytherapy trials, although there was a very slight trend towards increasing triangulation at day 4,5 and $10(p<0.2)$ (Supp. Figure 1C). Interestingly, instabilities were almost absent in this data set, when running nonparametric analysis on Poincare plots from the combination study (Figure $3 \mathbf{C}$ ) versus those for individual $\mathrm{HCQ}$ (significant, $\mathrm{p}<0.05$ ) or $\mathrm{AZM}$ (non significant trend) (Figure $3 \mathbf{A}, \mathbf{B}$ ). Although we observed a clear increase in $\mathrm{APD}_{80}$, and an increase of $\mathrm{CaD}_{80}$ above $600 \mathrm{~ms}$ at day 2 and day $4-10$ (Figure 3E), chronic polytherapy resulted in fewer arrhythmic events and only mild instability compared to monotherapy (Figure 3I). At most, 33\% of tissues showed arrhythmia at day 3 and 6 , with 16\% arrhythmias at day 4, 5, 7 and 9 (Figure 3F). Non-parametric contigency analysis showed a decrease between EAD instances in polytherapy versus HCQ (significant, $p<0.05$ ) or $A Z M$ (trend, $p<0.1$ ) monotherapy. No tissues had weak signal or stopped beating. At the pathophysiologic level, this data fit well with prior studies describing the important role of AP triangulation in the transition from benign AP prolongation to unstable repolarization[37].

Together, these observations suggest that polytherapy rescues arrhythmogenesis resulting from the individual drugs. Recent clinical studies demonstrated chronic exposure to combination of $\mathrm{HCQ}$ and AZM led to QT increases with few arrhytmia events [17, 35, 38]. The concordance of the cardiac MPS data to arrive at similar conclusions demonstrates its power in predicting cardiac liabilities for combination therapy of repurposed drugs to treat SARS-CoV-2. Mechanisms explaining how arrhythmic events are absent despite a significant increase of the QT interval, can be complex and additional studies would be required to elucidate HCQ and AZM polytherapy-dependent mechanisms. However, based on the fact that $\mathrm{HCQ}$ and $\mathrm{AZM}$ have known multichannel blocking effects, and that $I_{\mathrm{CaL}}$ and $I_{\mathrm{Na}}$ block is known to reduce $I_{K r}$ dependent arrhythmias[33], we can hypothesize that the combination of both drugs can synergistically increase multichannel block responsible for lower arrhythmic instances when compared to monotherapies. 


\section{Proteomics analysis of MPS effluent reveal candidate biomarkers for cardiotoxicity monitoring in patients treated with $H C Q$ and $A Z M$ :}

For the polytherapy pharmacology study, media were analyzed for over 92 proteins as biomarkers of tissue injury (Figure 4A). The proteomics analysis of the MPS effluent included a wide array of biomarkers, most of them measurable in patients' plasma, associated with different cardiac mechanisms, from morphology, cytoskeleton, mechanics to apoptosis and stress response. Cardiac troponin I (TNNI3), is a well known biomarker of cardiac injury and increased risk in mortality, common in COVID19 patients with underlying cardiovascular conditions[39]. Chronic exposure to the combination of AZM and $\mathrm{HCQ}$ showed no significant change in TNNI3 expression, suggesting that arrhythmic tissues are not undergoing major tissue damage. However, a clear decrease in erythropoietine (EPO) was observed. HPGDS, an intracellular enzyme that catalyzes the conversion of PGH2 to PGD2, was shown to decrease significantly. Interestingly, similar significant changes were also observed for carbonic anhydrase 14 (CA14) and tyrosine-protein kinase Fes/Fps (FES). These intracellular proteins are typically not secreted[40], and therefore are not strong biomarkers unless cells were damaged. The fact that alterations in levels for these proteins were observed in our study, suggests some degree of cell damage or stress, but not to the extent where troponin-actin complexes break down[41]. It is known that CA14 facilitates lactic acid transport across the cardiac sarcolemma [42], as well as improves myocardial energetics by facilitating mitochondria $\mathrm{CO}_{2}$ clearance [43]. We hypothesize the drug-related change in CA14 expression is a mechanism for the muscle to adapt antioxidant, contraction or waste management mechanisms to counter-balance cardiotoxic effects. Identification of biomarkers in the context of $\mathrm{HCQ}$ and $\mathrm{AZM}$, or other polytherapies, will be a valuable tool in the design of COVID-19 therapeutics trials.

\section{Summary}


The outcomes of this paper suggest that chronic drug exposure in this MPS format elicits arrhythmic outcomes similar to those observed in published clinical trials[17, 22, 23, 35, 36, 38]. Specifically, the known arrhythmia risk of $\mathrm{HCQ}$ and $\mathrm{AZM}$ alone was recapitulated by our in vitro observation of $\mathrm{APD}_{80}$ increase in combination with arrhythmic events. Combination therapy also exhibited an increase in QT, but compared to monotherapy, was benign at inducing arrhythmogenic behaviors. This also corresponds with recent clinical findings [38]. Together, these data suggest that this our high content in vitro heart muscle model can aid clinicians in clinical trial design, rapidly predict the cardiac outcomes of polytherapy for SARS-CoV-2 treatment, and help to identify relevant biomarkers to monitor during clinical trials for potential COVID-19 therapeutics.

\section{Limitations:}

Clinical QT interval values represent the summation of all the electrical activity in the ventricles. We used $\mathrm{APD}_{80}$ as a proxy for clinical QT prolongation, which is a credible and common approach to compare directional effects and provide some mechanistic insight, but is not sufficiently sensitive for precise prediction of instability thresholds [30]. COVID-19 patients with drug-induced QT interval changes of $>60 \mathrm{~ms}$ or QT interval values above $500 \mathrm{~ms}$ are considered high risk, and treatment is suspended[32]. Our cardiac muscle exhibited unphysiologically high $\mathrm{APD}_{80}$ values in response to chronic drug exposure. The mechanism for these large responses is unclear, but likely related to a combination of the hiPSC source, well-known modest maturity of hiPSC-CMs relative to the adult human heart, and possibly due to the altered current source-sink relationship in these very small tissues. Additionally, we have used a single patient line to perform this study, albeit with a significant number of replicates. By screening more patient lines one can achieve a clinically relevant dataset; although anticipated patient variability will require further expansion of the data size. In future work, this study can be extended to diseased cell lines to better understand the arrhythmic risk of patients with 
cardiovascular complications or comorbidities (i.e., diabetes) who are most likely to be seriously affected by SARS-CoV2.

\section{Study highlight}

What is the current knowledge on the topic?

As the global pandemic of COVID-19 has expanded, clinicians were pressed to treat patients with new drug combinations, in the absence of regulatory approval. Even ten months after the first cases, FDAapproved medicines for the treatment of COVID-19 are just emerging with mixed results. There is a need for tools to rapidly screen for cardiac liability associated with potential therapeutics.

What question did this study address?

Does Hydroxychloroquine and Azithromycin COVID-19 polytherapy show synergetic cardiac liability when compared to their monotherapy?

What does this study add to our knowledge?

In this in vitro safety 'clinical trial', hydroxychloroquine or azithromycin alone showed significant APD80 (proxy for QT) prolongation and arrhythmia, whereas their combination polytherapy rescued instances of arrhythmias while increasing APD80.

How might this change clinical pharmacology or translational science?

This study demonstrates that a complex in vitro tissue model (cardiac MPS) can predict arrhythmias and rhythm instabilities under experimental conditions mimicking safety clinical trials. We also identified biomarkers associated with cardiac injury, which can be used to design clinical trial monitoring protocols.

\section{Acknowledgements}


We thank Bruce Conklin (Gladstone Institutes, San Francisco, USA) for technical advice on the WTC iPSC line. We thank the Marvel Nanofabrication laboratory (UC Berkeley) and their staff for assistance and technical advices for microfabrication procedures.

\section{Author Contributions}

All authors participated in the study design, analysis of the data, interpretation of the results and review of the manuscript; $B C$ and VC conducted the main experiments and qualitative EAD characterization; BS helped with cell culture and MPS preparation; AGE helped with interpretation of electrophysiological recordings. HF developed the code to analyze action potential waveforms and Poincare plots; EM provided BeRST-1; BC, VC, AGE and KEH wrote the manuscript; BC prepared the figures and caption; KEH funded the work.

\section{Materials and Methods}

\section{Cardiomyocyte Differentiation}

The cardiomyocytes (CM) used in this study were derived from human induced pluripotent stem cells (hiPSC) via small molecular manipulation of the Wnt/ß-catenin signaling pathway [52] and were purified using glucose deprivation[53] to select for cardiomyocytes only.

\section{Fabrication and cell loading of Cardiac MPS}

The microfluidic design for each MPS consisted of 4 identical cell culture chambers (1300 by $130 \mu \mathrm{m})$ with media channels running parallel on either side of the cell culture chambers. The microfluidic devices were fabricated from Polydimethylsiloxane (PDMS) using classic replica molding techniques [24, 29]. Upon loading, 4000 lactate purified hiPSC-CM were injected into each tissue chamber. The following day and every other day from then on, media was changed to our in-house 'Maturation Media' (MM) as described in [29]. MPS tissues were allowed to mature for at least 10 days before any subsequent experiments were performed.

\section{Drug Preparation For Pharmacology Studies}


We based our study on the following protocols: Clinical drug administration for HCQ is $400 \mathrm{mg}$ twice per day followed by $200 \mathrm{mg}$ twice per day for 4 days. AZM is $500 \mathrm{mg}$ on day 1 followed by $250 \mathrm{mg}$ per day for the following 4 days. Based on clinical peak plasma concentration $\left(\mathrm{C}_{\max }\right)$ and area under curve for 24h[44][45], we exposed the MPS tissues to $0.24 \mu \mathrm{M} \mathrm{HCQ}$ at day 1 and $0.12 \mu \mathrm{M} \mathrm{HCQ}$ from day 2 to day $10 ;$ and to $2.67 \mu \mathrm{g}^{\star} \mathrm{h} / \mathrm{ml} / 24 \mathrm{~h}=0.111 \mu \mathrm{g} / \mathrm{ml}=0.15 \mu \mathrm{M} \mathrm{AZM}$ at day 1 and $0.056 \mu \mathrm{g} / \mathrm{ml}(0.075 \mu \mathrm{M})$ AZM at day 2 to day 10 .

Thorough Action Potential Analysis As A Proxy For Clinical QT Interval Study And Arrhythmia Prediction

Clinically, drug-induced QT prolongation is a strong predictor of arrhythmic cardiotoxicity in patients. At the cellular level, AP prolongation and increased AP triangulation indicate slowed repolarization and are strong markers of whole heart QT prolongation and arrhythmia [37], translated by observations of Early afterdepolarizations (EADs) [46-49], and subsequent Torsade de Pointes[46]. Large beat-to-beat variation in AP duration is a specific indicator of repolarization instability, which can be readily visualized by Poincare plots, plotting $\mathrm{CaD}_{80}$ of each $\left(\mathrm{n}^{\text {th }}\right)$ beat in the 30 second calcium recording, against $\mathrm{CaD}_{80}$ of the preceding beat $(\mathrm{n}-1)^{\text {th }}$, normalized to the $\mathrm{CaD}_{80}$ mean (Figure $\left.3 \mathbf{A}-\mathbf{C}\right)$. We also performed a qualitative and non-parametric evaluation of drug arrhythmogenesis by categorizing arrhythmic behaviors present in the calcium time-series (Figure 3D-F).

\section{Plasma Protein Profiling Using Olink Multiplex Panel}

Effluents were sent to Olink Proteomics for quantification of proteins associated with toxicity and tissue damage. Olink Proteomics uses multiplex proximity extension assay (PEA) panels [50]. In this study, we have used the Organ Damage panel which consist of 92 unique markers of toxicity and cellular damage. 


\section{Statistics}

All statistics were calculated using GraphPad Prism. All electrophysiology data were analyzed with one way ANOVA repeated measures and Dunnett's post-hoc correction with multiple comparison to day 0 and to one another was run. If some values were missing, mixed-effects model was run. Non parametric Chi-squared approximation was run for qualitative arrhythmic events assessment in a pairwise manner. Significance was determined with $p$-value $<0.05$.

More detailed information can be found in the supplementary information. 


\section{References}

1. Varga, Z., et al., Endothelial cell infection and endotheliitis in COVID-19. The Lancet, 2020. 395(10234): p. $1417-1418$.

2. Lu, Y., et al., Cerebral Micro-Structural Changes in COVID-19 Patients - An MRI-based 3-month Follow-up Study. EClinicalMedicine, 2020. 25: p. 100484.

3. Fried, J.A., et al., The Variety of Cardiovascular Presentations of COVID-19. Circulation, 2020. 141(23): p. 1930-1936.

4. Farouk, S.S., et al., COVID-19 and the kidney: what we think we know so far and what we don't. Journal of Nephrology, 2020.

5. A Pérez-Bermejo, J., et al., SARS-CoV-2 infection of human iPSC-derived cardiac cells predicts novel cytopathic features in hearts of COVID-19 patients. 2020, Cold Spring Harbor Laboratory.

6. Arun Sharma, et al., Human iPSC-Derived Cardiomyocytes are Susceptible to SARS-CoV-2 Infection. 2020, Cold Spring Harbor Laboratory.

7. Diana Lindner, et al., Association of Cardiac Infection With SARS-CoV-2 in Confirmed COVID-19 Autopsy Cases. JAMA Cardiology, 2020.

8. Gautret, P., et al., Hydroxychloroquine and azithromycin as a treatment of COVID-19: results of an openlabel non-randomized clinical trial. Int J Antimicrob Agents, 2020: p. 105949.

9. Yao, X., et al., In Vitro Antiviral Activity and Projection of Optimized Dosing Design of Hydroxychloroquine for the Treatment of Severe Acute Respiratory Syndrome Coronavirus 2 (SARS-CoV-2). Clin Infect Dis, 2020.

10. Wang, M., et al., Remdesivir and chloroquine effectively inhibit the recently emerged novel coronavirus (2019-nCoV) in vitro. Cell Res, 2020. 30(3): p. 269-271.

11. Huang, M., et al., Treating COVID-19 with Chloroquine. J Mol Cell Biol, 2020.

12. Xueting Yao, et al., In Vitro Antiviral Activity and Projection of Optimized Dosing Design of Hydroxychloroquine for the Treatment of Severe Acute Respiratory Syndrome Coronavirus 2 (SARS-CoV2). Clinical Infectious Diseases, 2020. 71(15): p. 732-739.

13. Arshad, S., et al., Treatment with hydroxychloroquine, azithromycin, and combination in patients hospitalized with COVID-19. International Journal of Infectious Diseases, 2020. 97: p. 396-403.

14. Lagier, J.-C., et al., Outcomes of 3,737 COVID-19 patients treated with hydroxychloroquine/azithromycin and other regimens in Marseille, France: A retrospective analysis. Travel Medicine and Infectious Disease, 2020. 36: p. 101791.

15. Molina, J.M., et al., No Evidence of Rapid Antiviral Clearance or Clinical Benefit with the Combination of Hydroxychloroquine and Azithromycin in Patients with Severe COVID-19 Infection. Médecine et Maladies Infectieuses, 2020.

16. Gérard, A., et al., "Off-label" use of hydroxychloroquine, azithromycin, lopinavir-ritonavir and chloroquine in COVID-19: A survey of cardiac adverse drug reactions by the French Network of Pharmacovigilance Centers. Therapies, 2020.

17. Rosenberg, E.S., et al., Association of Treatment With Hydroxychloroquine or Azithromycin With InHospital Mortality in Patients With COVID-19 in New York State. JAMA, 2020.

18. Furtado, R.H.M., et al., Azithromycin in addition to standard of care versus standard of care alone in the treatment of patients admitted to the hospital with severe COVID-19 in Brazil (COALITION II): a randomised clinical trial. The Lancet, 2020. 396(10256): p. 959-967.

19. Meyerowitz, E.A., et al., Rethinking the role of hydroxychloroquine in the treatment of COVID-19. The FASEB Journal, 2020. 34(5): p. 6027-6037.

20. Jankelson, L., et al., QT prolongation, torsades de pointes, and sudden death with short courses of chloroquine or hydroxychloroquine as used in COVID-19: A systematic review. Heart Rhythm, 2020. 
21. Ray, W.M., K. ; Hall, K.; Arbogast, P.; Stein, M., Azithromycin and the Risk of Cardiovascular Death. The New England Journal of Medicin, 2012.

22. Bessière, F., et al., Assessment of QT Intervals in a Case Series of Patients With Coronavirus Disease 2019 (COVID-19) Infection Treated With Hydroxychloroquine Alone or in Combination With Azithromycin in an Intensive Care Unit. JAMA Cardiology, 2020.

23. Chorin, E., et al., QT Interval Prolongation and Torsade De Pointes in Patients with COVID-19 treated with Hydroxychloroquine/Azithromycin. Heart Rhythm, 2020.

24. Mathur, A., et al., Human iPSC-based cardiac microphysiological system for drug screening applications. Sci Rep, 2015. 5: p. 8883.

25. Nunes, S.S., et al., Biowire: a platform for maturation of human pluripotent stem cell-derived cardiomyocytes. Nat Methods, 2013. 10(8): p. 781-7.

26. Loskill, P., et al., muOrgano: A Lego(R)-Like Plug \& Play System for Modular Multi-Organ-Chips. PLoS One, 2015. 10(10): p. e0139587.

27. Si, L., et al., Human organs-on-chips as tools for repurposing approved drugs as potential influenza and COVID19 therapeutics in viral pandemics. 2020.

28. Tang, H., et al., Human Organs-on-Chips for Virology. Trends in Microbiology, 2020.

29. Huebsch, N., et al., Metabolically-Driven Maturation of hiPSC-Cell Derived Heart-on-a-Chip. 2020, Cold Spring Harbor Laboratory.

30. Blinova, K., et al., International Multisite Study of Human-Induced Pluripotent Stem Cell-Derived Cardiomyocytes for Drug Proarrhythmic Potential Assessment. Cell Reports, 2018. 24(13): p. 3582-3592.

31. Yang, Z., et al., Azithromycin Causes a Novel Proarrhythmic Syndrome. Circulation: Arrhythmia and Electrophysiology, 2017. 10(4): p. e003560.

32. Asensio, E., et al., Recommendations for the measurement of the QT interval during the use of drugs for COVID-19 infection treatment. Updatable in accordance with the availability of new evidence. Journal of Interventional Cardiac Electrophysiology, 2020.

33. Blinova, K., et al., Comprehensive Translational Assessment of Human-Induced Pluripotent Stem Cell Derived Cardiomyocytes for Evaluating Drug-Induced Arrhythmias. Toxicol Sci, 2017. 155(1): p. 234-247.

34. Sharma, T.S., et al., Hydroxychloroquine Use Is Associated With Decreased Incident Cardiovascular Events in Rheumatoid Arthritis Patients. Journal of the American Heart Association, 2016. 5(1): p. e002867.

35. Vouri, S.M., T.N. Thai, and A.G. Winterstein, An evaluation of co-use of chloroquine or hydroxychloroquine plus azithromycin on cardiac outcomes: A pharmacoepidemiological study to inform use during the COVID19 pandemic. Research in Social and Administrative Pharmacy, 2020.

36. Chorin, E., et al., The QT Interval in Patients with SARS-CoV-2 Infection Treated with Hydroxychloroquine/Azithromycin. 2020.

37. M. Hondeghem, L., L. Carlsson, and G. Duker, Instability and Triangulation of the Action Potential Predict Serious Proarrhythmia, but Action Potential Duration Prolongation Is Antiarrhythmic. Circulation, 2001. 103(15): p. 2004-2013.

38. Cipriani, A., et al., Arrhythmic profile and 24-hour QT interval variability in COVID-19 patients treated with hydroxychloroquine and azithromycin. International Journal of Cardiology, 2020.

39. Sandoval, Y., J.L. Januzzi, and A.S. Jaffe, Cardiac Troponin for Assessment of Myocardial Injury in COVID19. Journal of the American College of Cardiology, 2020. 76(10): p. 1244-1258.

40. F Pontén, K.J., M Uhlen, The Human Protein Atlas--a tool for pathology The journal of pathology, 2008. 216(4): p. 387-393.

41. Luciano Babuin, A.S.J., Troponin: the biomarker of choice for the detection of cardiac injury. CMAJ, 2005. 173(10).

42. Hallerdei, J., et al., T Tubules and Surface Membranes Provide Equally Effective Pathways of Carbonic Anhydrase-Facilitated Lactic Acid Transport in Skeletal Muscle. PLoS ONE, 2010. 5(12): p. e15137. 
43. Marie A. Schroedera, M.A.A., Alzbeta Hulikovaa, Claudiu T. Supuranb, Kieran Clarkea,Richard D. Vaughan-Jonesa, Damian J. Tylera, and Pawel Swietacha, Extramitochondrial domain rich in carbonic anhydraseactivity improves myocardial energetics. PNAS physiology, 2013.

44. Drugbank, Hydroxychloroquine, https://www.drugbank.ca/drugs/DB01611.

45. Liu, P., et al., Comparative pharmacokinetics of azithromycin in serum and white blood cells of healthy subjects receiving a single-dose extended-release regimen versus a 3-day immediate-release regimen. Antimicrob Agents Chemother, 2007. 51(1): p. 103-9.

46. Zhilin Qu , L.-H.X., Riccardo Olcese, Hrayr S Karagueuzian, Peng-Sheng Chen, Alan Garfinkel, James N Weiss, Early afterdepolarizations in cardiac myocytes: beyond reduced repolarization reserve Cardiovascular Research, 2013. 99(1): p. 6-15.

47. Diana X Tran, D.S., Arik Yochelis, James N Weiss, Alan Garfinkel, Zhilin Qu, Bifurcation and chaos in a model of cardiac early afterdepolarizations Physical Review Letter, 2009. 102(25).

48. Daisuke Sato, L.-H.X., Ali A. Sovari, Diana X. Tran, Norishige Morita, Fagen Xie, Hrayr Karagueuzian, Alan Garfinkel, James N. Weiss, and Zhilin Qu, Synchronization of chaotic early afterdepolarizations in the genesis of cardiac arrhythmias. PNAS, 2009. 106(9): p. 2893-2988.

49. Yuanfang Xie , G.H., Daisuke Sato, James N Weiss, Alan Garfinkel, Zhilin Qu, Dispersion of refractoriness and induction of reentry due to chaos synchronization in a model of cardiac tissue Physical Review Letter, 2007. 99(11).

50. Erika Assarsson, et al., Homogenous 96-Plex PEA Immunoassay Exhibiting High Sensitivity, Specificity, and Excellent Scalability. PLOS ONE, 2014. 


\section{Figure Legends}

Figure 1. The cardiac microphysiological system. (A) Photograph of a cardiac MPS in fluorescent light with feeding tubing. (B) Brightfield image of a cardiac MPS loaded with 4,000 human induced pluripotent stem cell-derived cardiomyocytes. The cell chamber is separated from adjacent feeding channels via a fenestration barrier of $2 \mu \mathrm{m}$ wide grooves allowing for nutrient diffusion while protecting the tissue in the cell chamber from media flow-induced shear stress. The anchoring pillars on either side of the cell chamber help keep the heart muscle elongated and provide resistance for contraction. (C) Representative images of the same tissue under GFP fluorescence for calcium transient recordings (top) or FarRed voltage dye staining (bottom). (D) The top graph shows a typical ECG recording from which the clinical QT interval can be determined. We use $\mathrm{APD}_{80}$ as a proxy for QT duration, corresponding to the duration of the action potential at $80 \%$ of its repolarization (bottom). The APD (red) and Ca (green) waveforms are timestamped identifying temporal kinetics.

Figure 2. Electrophysiology analysis of chronic exposure to hydroxychloroquine (HCQ), azithromycin (AZM) or their polytherapy. Doses were chosen to closely mimic clinical trial drug prescription used for COVID-19 treatment: $0.24 \mu \mathrm{M} \mathrm{HCQ}$ and $0.15 \mu \mathrm{M}$ AZM on day1 followed by $0.12 \mu \mathrm{M}$ and $0.075 \mu \mathrm{M}$ AZM on day 2 to day 10. Polytherapy was the combination of both monotherapy doses (Supp. Table 1). Violin plots demonstrate spontaneous beating during the therapy for HCQ (A), AZM (C) or polytherapy (E). Violin plots depicting the change in $\mathrm{APD}_{80}$ during the therapy for $\mathrm{HCQ}(\mathbf{B}), \mathrm{AZM}$ (D) or polytherapy $(\mathbf{F})$. APD 80 values were calculated from spontaneous recording, corrected for beat rate using the Fredericia correction[1]. In all graphs, the values were normalized to baseline at day 0 for each tissue. Each point corresponds to one heart muscle. The violin plots show the arithmetic median (solid line) and upper and lower quartile (dashed lines) as well as minimum and maximum values (truncation of violin shape). All tissues analyzed were within inclusion criteria of $\mathrm{APD}_{80}<500 \mathrm{~ms}$ at baseline. Statistics run were one-way ANOVA repeated measures with multiple comparison to baseline day 0 and Dunnett's post-hoc correction. ${ }^{*} p<0.05 ;{ }^{* *} p<0.01 ;{ }^{* *} p<0.001$. 
Figure 3. Instability and arrhythmic study of chronic exposure to hydroxychloroquine (HCQ), azithromycin (AZM) and their polytherapy. Poincare plots[2] were used to visualize rhythm instabilities in different tissues. Where small cluters of daily traces represent minimal arrhythmic risk, and large complex polygons are indicative of rhythm instability and high arrhythmic risk. (A) Representative Poincare graph of a tissue exposed to $\mathrm{HCQ}$ only. Disorganized polygons can be observed at day 5 and 10 indicative of drug-indueced arrhythmia. (B) Poincare graph of AZM-treated heart muscle where instabilities were observed starting on day 6. (C) Representative graph of polytherapy instabilities, which show fewer and smaller polygons when compared to monotherapy, indicative of reduced arrhythmic risk. (D-F) Histograms showing weak or no signal (black), $\mathrm{CaD}_{80}$ above $600 \mathrm{~ms}$ with (striated) or without (grey) EAD and arrhythmic event (pink) as percentage of total heart muscles (i.e., MPS). The analysis was performed for $\mathrm{HCQ}$ alone (D), AZM alone (E), and for polytherapy $(\mathbf{F})$. Representative calcium transient trace at baseline and after exposure to 9 days of HCQ (G) or 10 days of AZM (H) where EAD instances can be observed (red arrows). (I) Representative calcium transient trace at 10 day exposure polytherapy showing no EAD.

Figure 4. Proteomics analysis of microphysiological systems effluent during chronic exposure to $\mathrm{HCQ}$ and $\mathrm{AZM}$ polytherapy. Scatter plot of biomarkers showing significant changes with increasing doses of polytherapy in chronic study. The effluent media was analyzed on day 2, 4, 6, 8 and 10. In the data post-processing, we only included experiments with $>65 \%$ of samples above limit of detection. The limit of detection is set at 3 standard deviation above negative control values. Statistics run were one-way ANOVA with multiple comparison to one another and Dunnett's post-hoc correction. * $p<0.05$; ${ }^{* *} \mathrm{p}<0.01$. TNNI3 (cardiac troponin I), EPO (Erythropoietine), HPGDS (Hematopoietic prostaglandin D synthase), CA14 (Carbonic anhydrase 14), FES (FES proto-oncogene, Tyrosine Kinase)

1. Vandenberk, B., et al., Which QT Correction Formulae to Use for QT Monitoring? Journal of the American Heart Association, 2016. 5(6): p. e003264.

2. J N Weiss, A.G., H S Karagueuzian, Z Qu, P S Chen, Chaos and the transition to ventricular fibrillation: $a$ new approach to antiarrhythmic drug evaluation. Circulation, 1999. 99(21): p. 2819-2826. 
bioRxiv preprint doi: https://doi.org/10.1101/2020.12.21.423869; this version posted December 28, 2020. The copyright holder for this preprint (which was not certified by peer review) is the author/funder. All rights reserved. No reuse allowed without permission.

\section{Supplementary Information Titles :}

\section{Supplemental Materials and Methods}

\section{Supplemental Figure Legends}

\section{Supplemental Tables}


bioRxiv preprint doi: https://doi.org/10.1101/2020.12.21.423869; this version posted December 28, 2020. The copyright holder for this preprint A (which was not certified by peer review) is the author/funder. All rights reserved. No 0 euse allowed without permission.

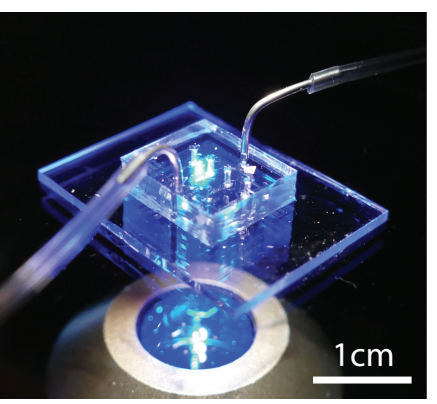

B

lood vessel-like channels

for nutrient and drug delivery and biomarker recovery

Anchoring pillars

for tissue support

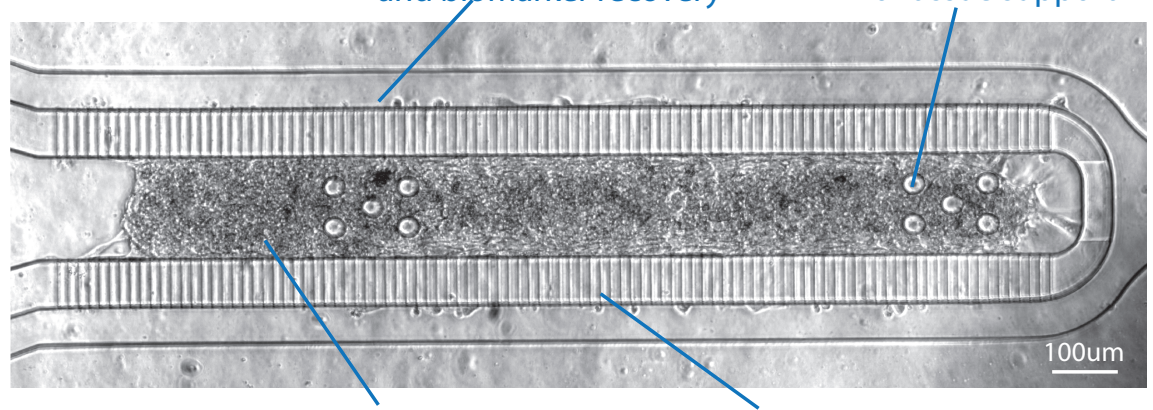

Micro-heart muscle

C

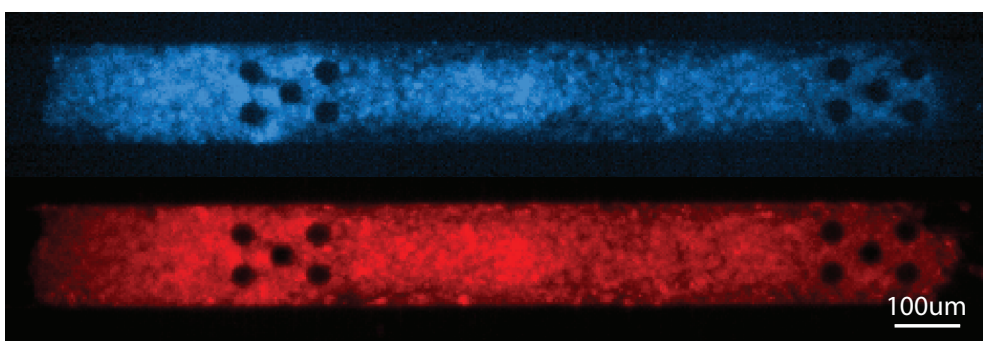

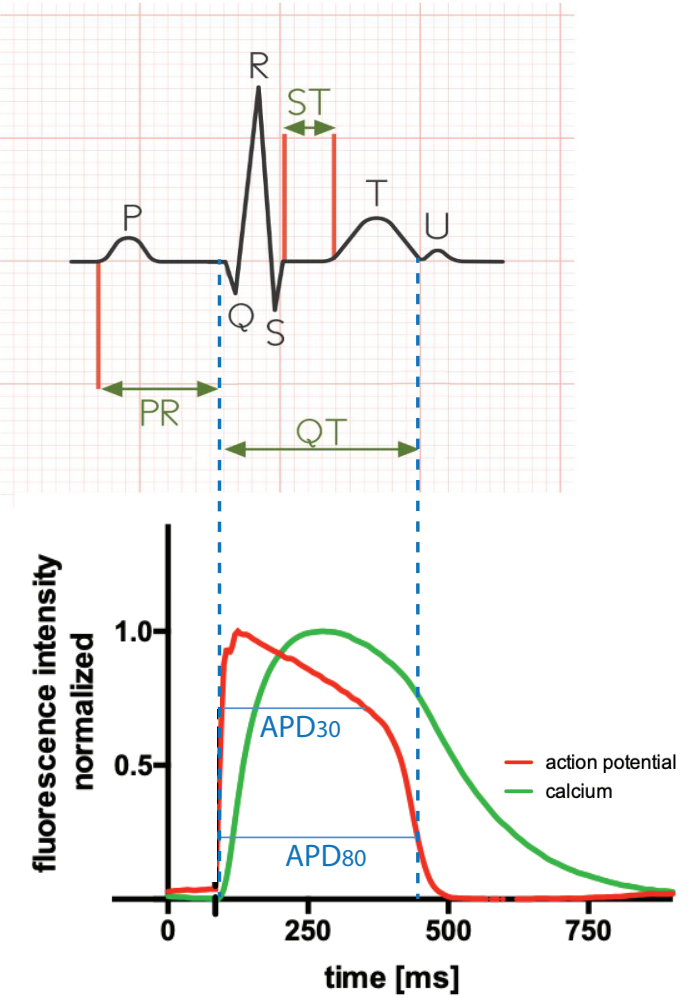


bioRxiv preprint doi: https://doi.org/10.1101/2020.12.21.423869; this version posted December 28, 2020. The copyright holder for this preprint A (which was not certified by peer review) is the author/funder. All righs reserved. No reuse allowed without permission.
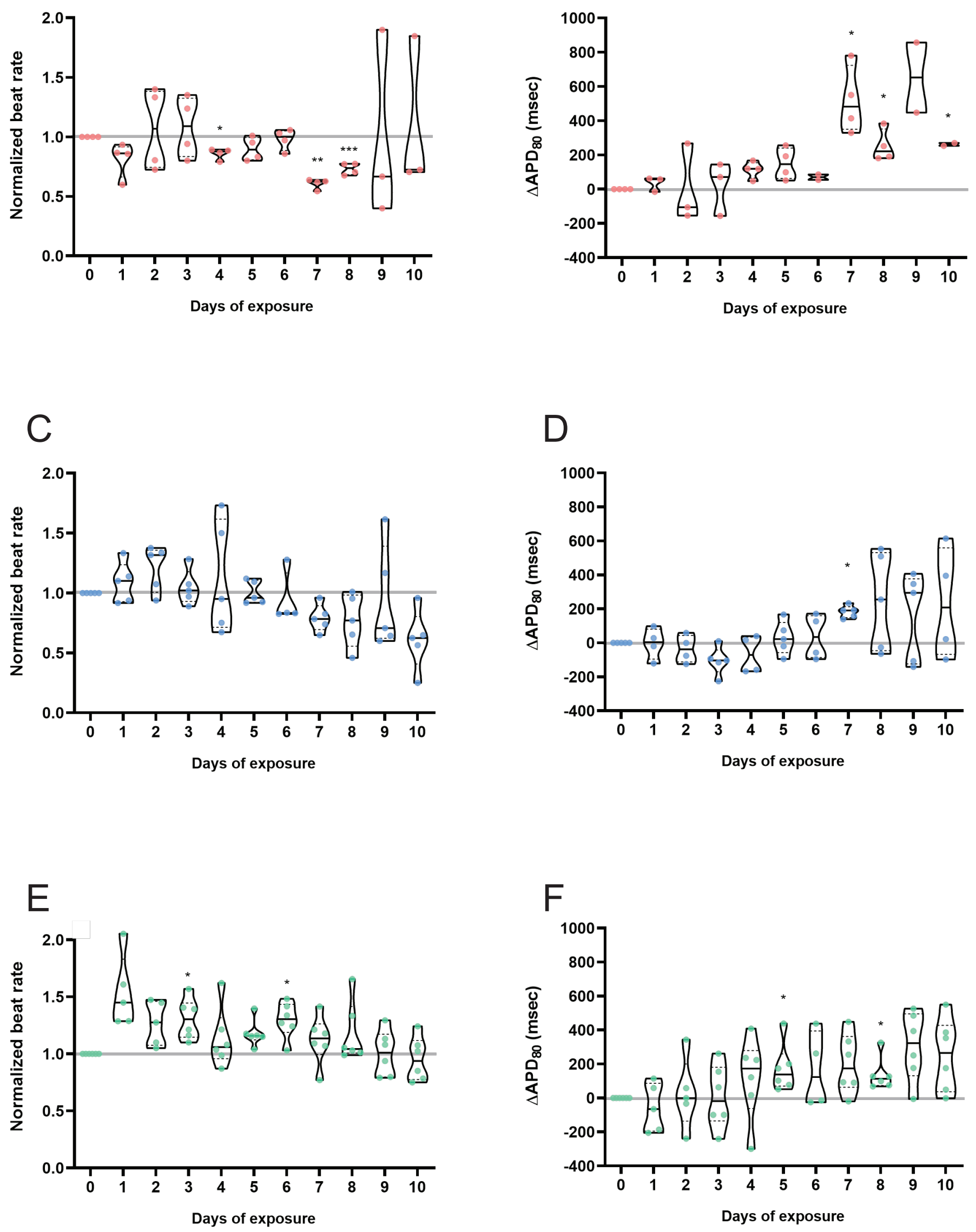
bioRxiv preprint doi: https://doi.org/10.1101/2020.12.21.423869; this version posted December 28, 2020. The copyright holder for this preprint (which was not certified by peer review) is the author/funder. All rights reserved. No reuse allowed without permission.
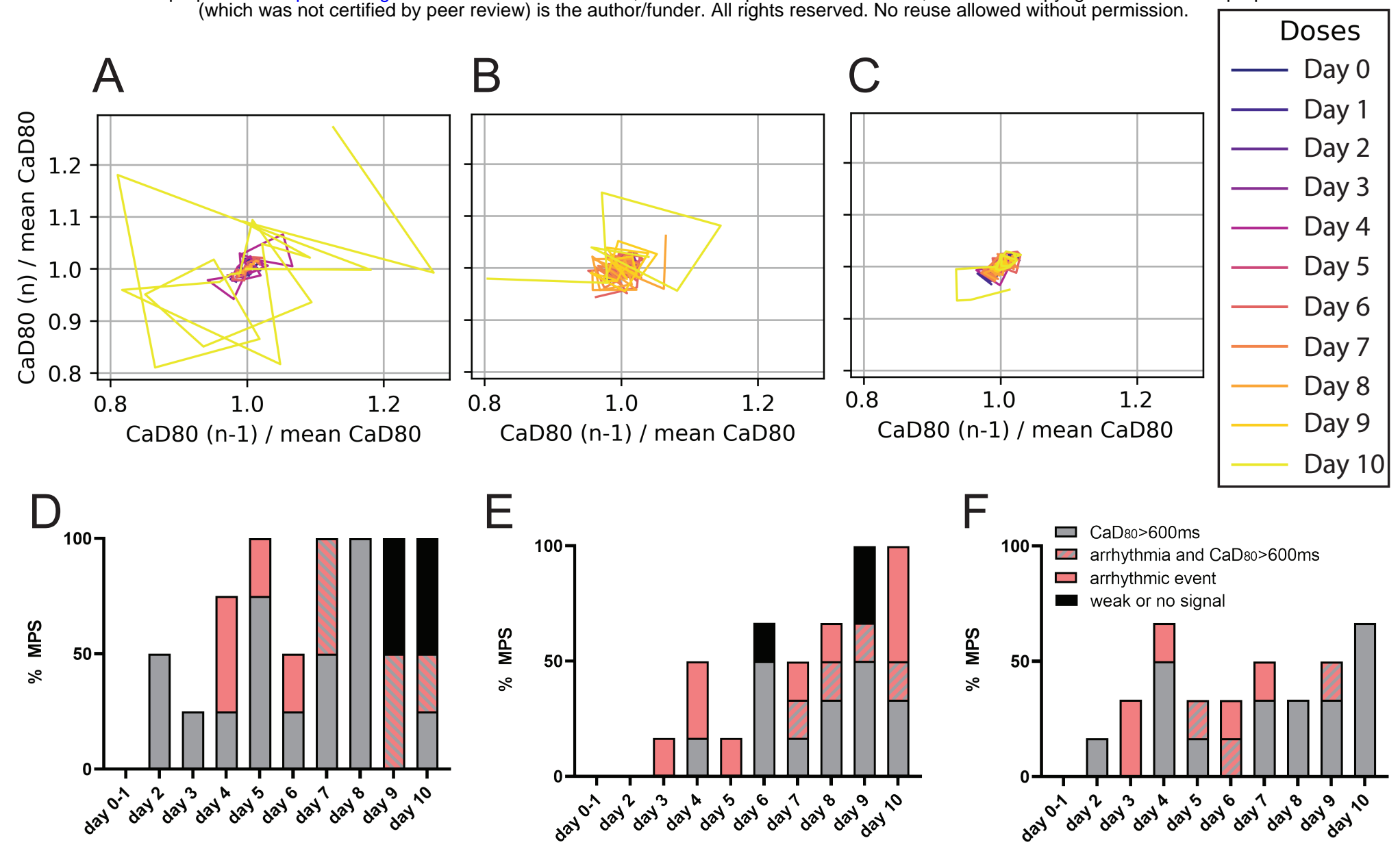

G
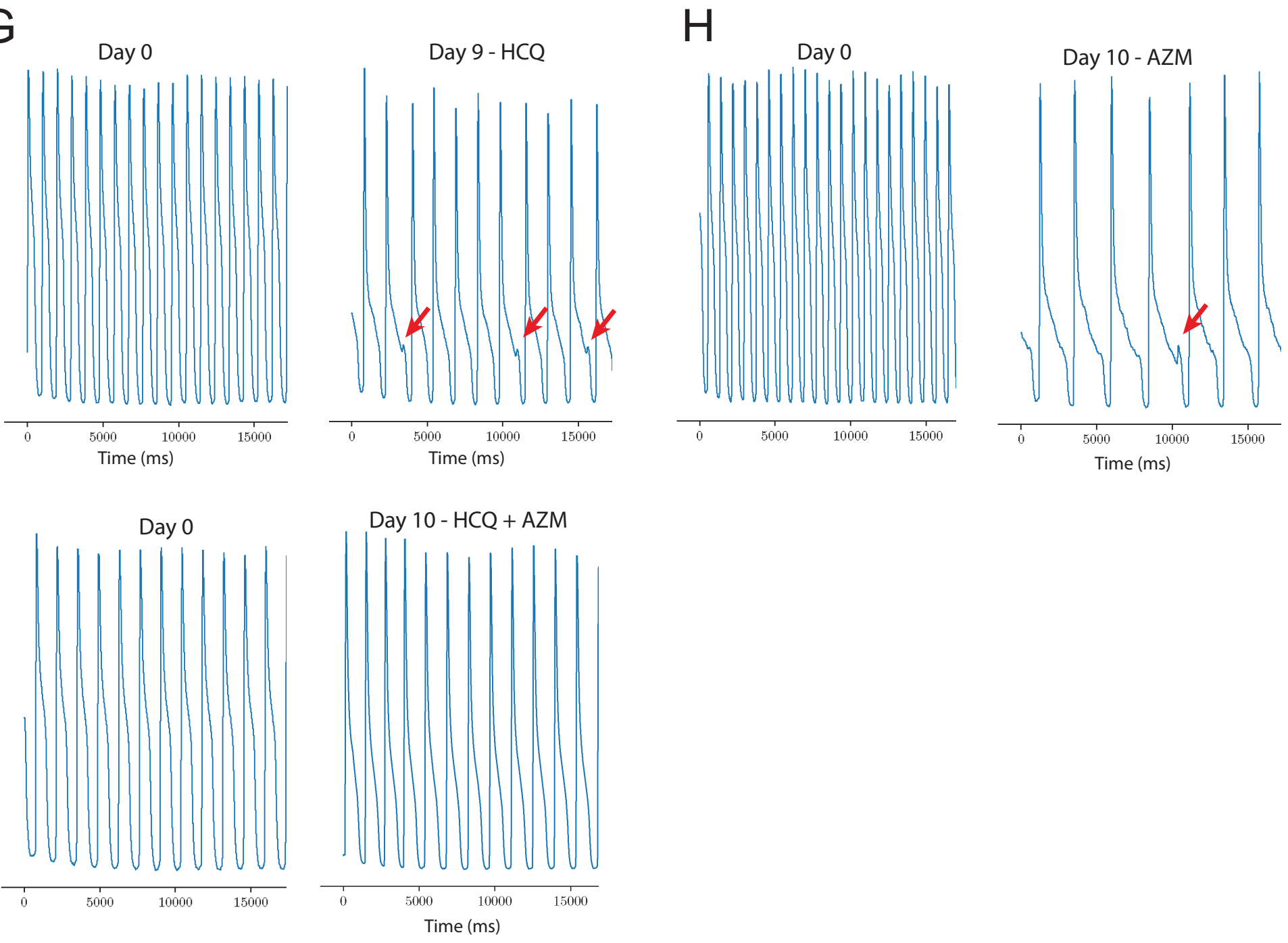


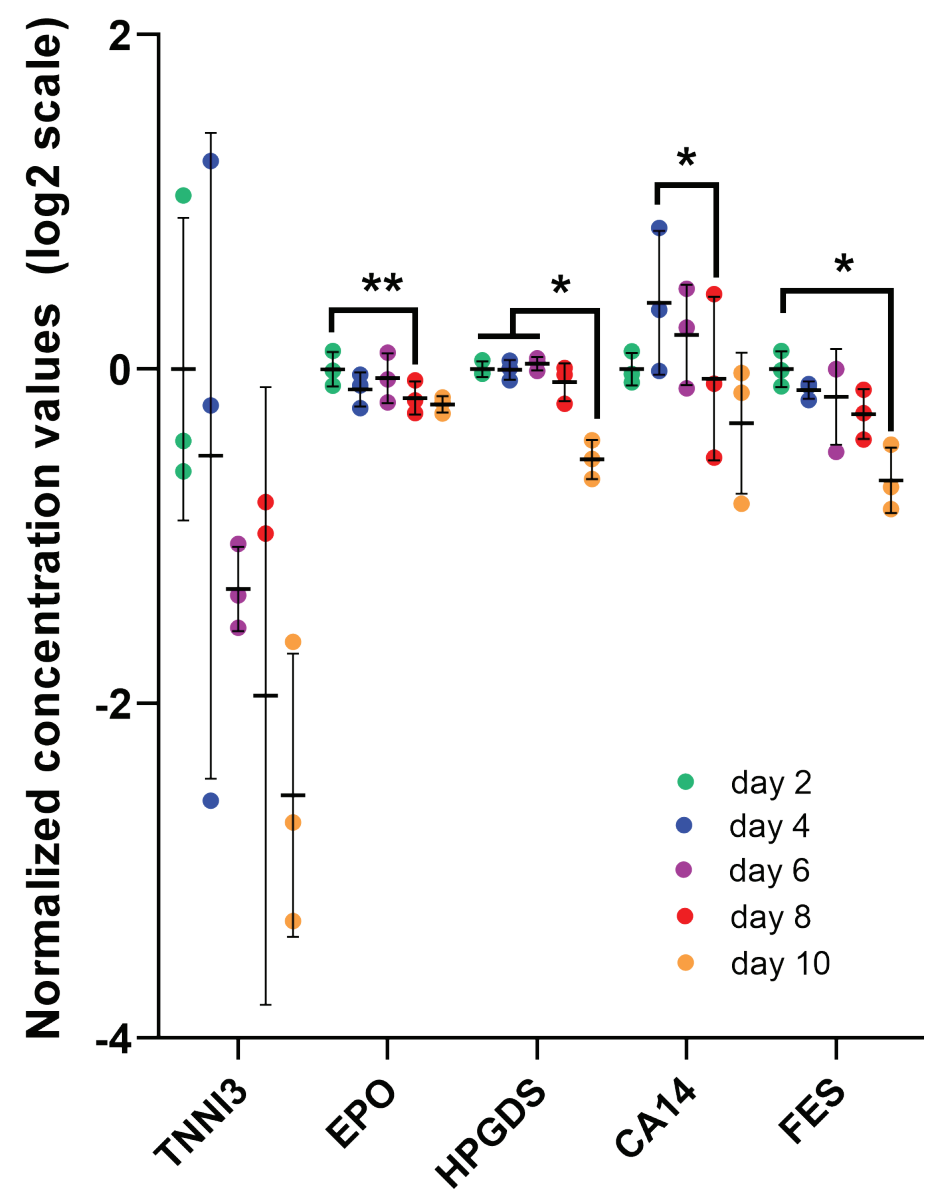

